\title{
Proportional valve with a piezoelectric actuator
}

\author{
Pawel Andrzej Laski ${ }^{1, *}$ \\ ${ }^{1}$ Kielce University of Technology, Faculty of Mechatronics and Mechanical Engineering, Department of Automation and Robotics, \\ Aleja Tysiaclecia Panstwa Polskiego 7, 25-314 Kielce, Poland
}

\begin{abstract}
The article concerns a slotted proportional valve for use in pneumatic and hydraulic systems. There is a growing demand for both hydraulic and pneumatic ultrafast proportional valves. The conducted analysis of literature confirms the lack of such solutions for proportional valves. The currently used pneumatic systems for selection and segregation of parts and objects require ultrafast valves. The presented solution for the proportional valve can significantly improve and accelerate this type of technological processes. Furthermore, fast proportional valves can be successfully used for positional control of pneumatic and hydraulic drives. The article presents the design of a slotted divide valve and sets the maximum mass flow rate for service roads.
\end{abstract}

\section{Introduction}

Currently, the series and large series production requires the removal of defective or damaged parts and objects from the production line without turning them off. One of the methods applied to achieve such an effect is blowing out an object moving in front of the nozzle arrangement by a strong air flow [1,2]. The inclusion and exclusion of air flow should be done in the shortest possible time and the value of flow rate should be tailored to the size and mass of the object $[3,4]$.

The nozzle arrangement is most frequently controlled by spool valves. Their task is to separate the air stream between flow windows of the valve and directing the flow of air in the direction of the object. Separation of the air stream takes place through suitably shaped edges of the valve spool and its body. Such a solution has several advantages, but also one drawback associated with the mass of the valve spool that causes a relatively long switching time. A new solution was proposed in order to shorten the response time and improve the parameters of classic valves. The valve design uses an ultrafast piezoelectric actuator with mechanical amplifier which guarantees a proportional shift of elements that separate the stream and thus the controlled flow of air. This resulted in a design of the proportional valve type 2/2 (two-way, two-position). The design constructed with the use of CFD software required the determination and presentation of a vector velocity field and pressure distribution in the cross-sections of flow windows $[5,6]$. The maximum flow values for the valve spool were determined on the basis of simulation studies. Based on simulation studies, it can be concluded that it is possible to build a proportional pneumatic valve with high values of the flow rate.

\section{Actuators used in valves.}

There are two types of piezoelectric actuators used in industrial and prototypical valves to control the fluid systems [7]. One of the solutions is a piezoelectric actuator in the form of a plate with thin thickness restrained to the body by one end, while the second end of the piezoelectric actuator opens or closes the flow window. In this solution the piezoelectric actuator moves at a high frequency with significant displacement of a few millimetres but the resulting force does not exceed a few Newtons [8]. The valves of this structure are used in preliminary stages to control the pressure relief valve and divide valve. A similar structure solution is used in a valve wherein the control element has a discoidal shape with a small thickness in relation to the diameter. As a result of deformation within the piezoelectric actuator, the central portion of the disc moves thus obtaining an opened flow window of the valve. Another solution that uses piezoelectric actuators involves structures in which the piezoelectric actuator has the form of a multi-layered stack. It uses structures in which piezoelectric actuators in the form of a stack open and close the flow windows, but in that case the movement of the propulsion system does not exceed $100 \mu \mathrm{m}$ [9]. A slight movement hinders the structure of valves in a significant way. This solution was used in injectors and unit pump injectors of diesel engines. Currently, researchers are trying to develop valve designs with multi-layered piezoelectric stacks, where slight movement of the piezoelectric actuator in the form of a stack is multiplied by mechanical levers and cams. This results in a significant movement of the whole drive, but decreases the force and operating frequency. The drive and control component in the designed structure of the valve is a multi-layered piezoelectric actuator with a mechanical amplifier in the form of an elliptical bracket. The displacement of drive

*Corresponding author: pawell@tu.kielce.pl 
amounts to $500 \mu \mathrm{m}$, the maximum locking force amounts to $570 \mathrm{~N}$ and the operating frequency is up to $460 \mathrm{~Hz}$. The multi-layered actuator of the valve in the form of a piezoelectric stack allows for the ultrafast movement of separation inserts. That is how the valve is opened and closed.

\subsection{Operation of a slotted divide valve type $2 / 2$}

The designed flow valve and distribution of the air stream is carried out by a suitably shaped assemblies that form flow windows. Such a solution lacks the typical separation slider. The operation of valves was shown in figure 1 and figure 2.

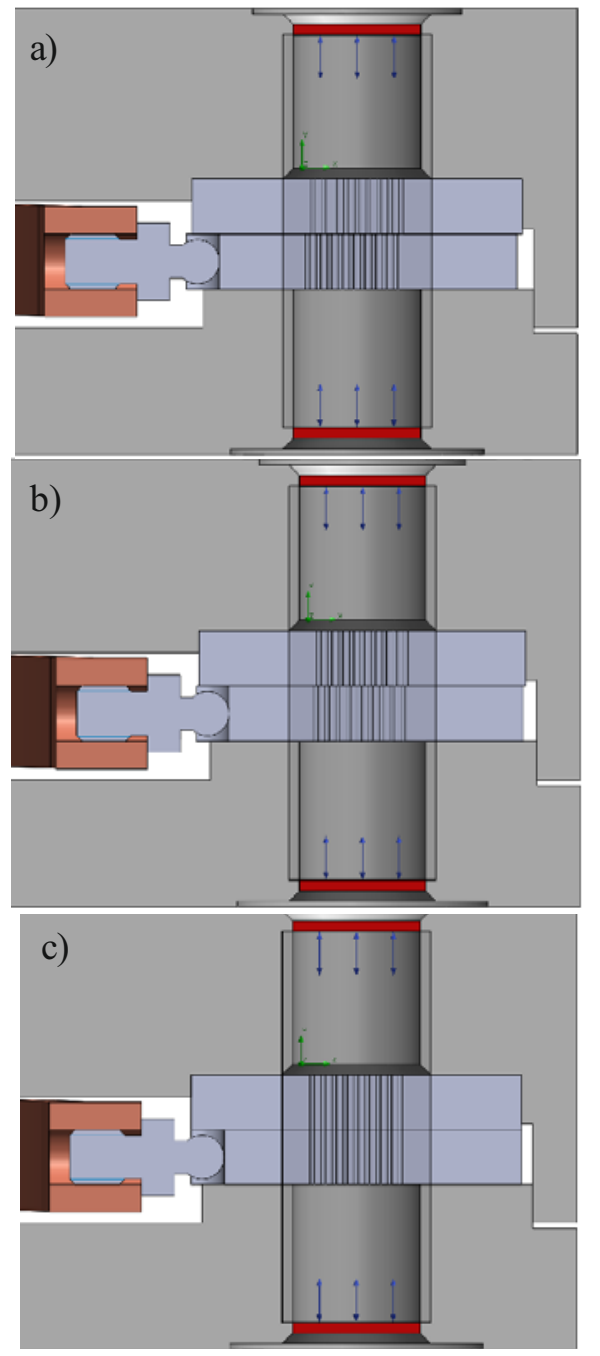

Fig. 1. A fragment of the cross section of the $5 / 3$ valve. a) neutral position - closed valve, b) $50 \%$ displacement of the actuator, c) the maximum flow - open position.

The present valve contains a singular slot in the form of a rectangle with one side equal to the displacement of a piezoelectric actuator. Whereas the size of the sides of a flow window in the form of a rectangular is adjusted to the designed flow rate and the total amount of slots. The discharge window of the valve accumulates the flows from individual slots and the obtained nominal flow of the valve. The number of slots corresponds to the surface of the target flow window for the path of $2 / 2$ valve. The valve comprises a flow path corresponding to the supply port 1 of the flow 2 , in the indicated body $(6,7)$ which are embedded in the slotted core plates (5) and corresponding groups of slots of the lower insert. The lower slotted insert is treated separately from one of the arms (4) of the amplifier. The second arm of the amplifier is attached to the body (6) of the valve by a screw. In a neutral position (figure 1a) the flow paths of the valve are completely closed. Figure $1 \mathrm{~b}$ shows the valve at $50 \%$ of the flow corresponding to the displacement of the insert with slots of $250 \mu \mathrm{m}$. Figure 1c shows the end position of the slotted core corresponding to the valve in the open position. The piezoelectric actuator enables the movement of inserts with slots in a proportionate manner, the lower plate insert can occupy intermediate position relative to the upper plate insert and the flow through flow paths will depend on the degree of slots opening.

The total area of the slots, creating a flow window, is equal to the surface of the valve connection G1/4" $(\sim 34 \mathrm{~mm} 2)$. An increase of the flow window surface does not constitute a technical problem for the designed structure. The flow window may be increased by adding more slots while maintaining the displacement of piezoelectric actuators equal to $500 \mu \mathrm{m}$. Figure 2 shows a three-dimensional model of the valve with a partial semi-transparent view.

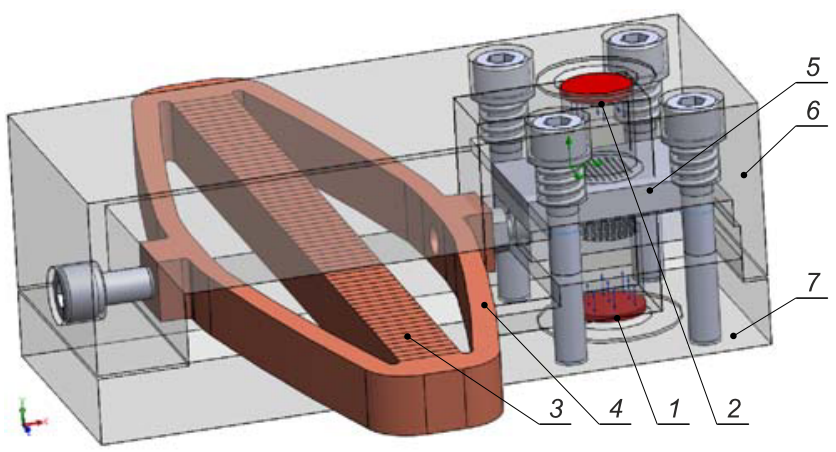

Fig. 2. Solid model of the valve. 1,2 - connections, 3 piezoelectric actuator, 4 - mechanical amplifier of the displacement, 5 - slotted separation inserts, 6,7 - valve body.

\section{Flow simulation (CFD)}

Figure 4 shows the results of simulation studies in the CFD ANSYS software. A spatial computing grid with irregular structure was set in order to conduct the analysis. The crucial area of the set grid was a group of slots where the minimum mesh size was $1 \mu \mathrm{m}$. It was assumed that the valve was used to control gases, including compressed filtered air with a maximum solid particle size not exceeding $40 \mu \mathrm{m}$. For the purpose of the study we assumed working pressure of the valve ranging from 0.25 to $0.7 \mathrm{MPa}$ and operating temperature ranging from $-10 \div+60{ }^{\circ} \mathrm{C}$. The CFD ANSYS software used in the modelling process uses a mathematical model describing the physics of flow for the turbulent motion of viscous fluid described by Reynolds equations and equations of stream continuity. The equations form the 
basis of an interrelationship, allowing the determination of pressure distribution and velocity field of the flow. The software was used to develop a computing grid, where the number of nodes of the analysed volume of fluid (air) was 283280 which corresponds to 1318183 components. Figure 3 shows the grid and the enlarged fragment within slots of the flow window. The following physical quantities were assumed for calculations: the ambient temperature $\quad T=20^{\circ} \mathrm{C}, \quad$ density $\rho=1.185 \mathrm{~kg} / \mathrm{m}^{3}$, supply pressure $0.6 \mathrm{MPa}$, reference pressure $p=101325 \mathrm{~Pa}$. Fig. 4, 5 shows the results of simulation research in the CFD software.
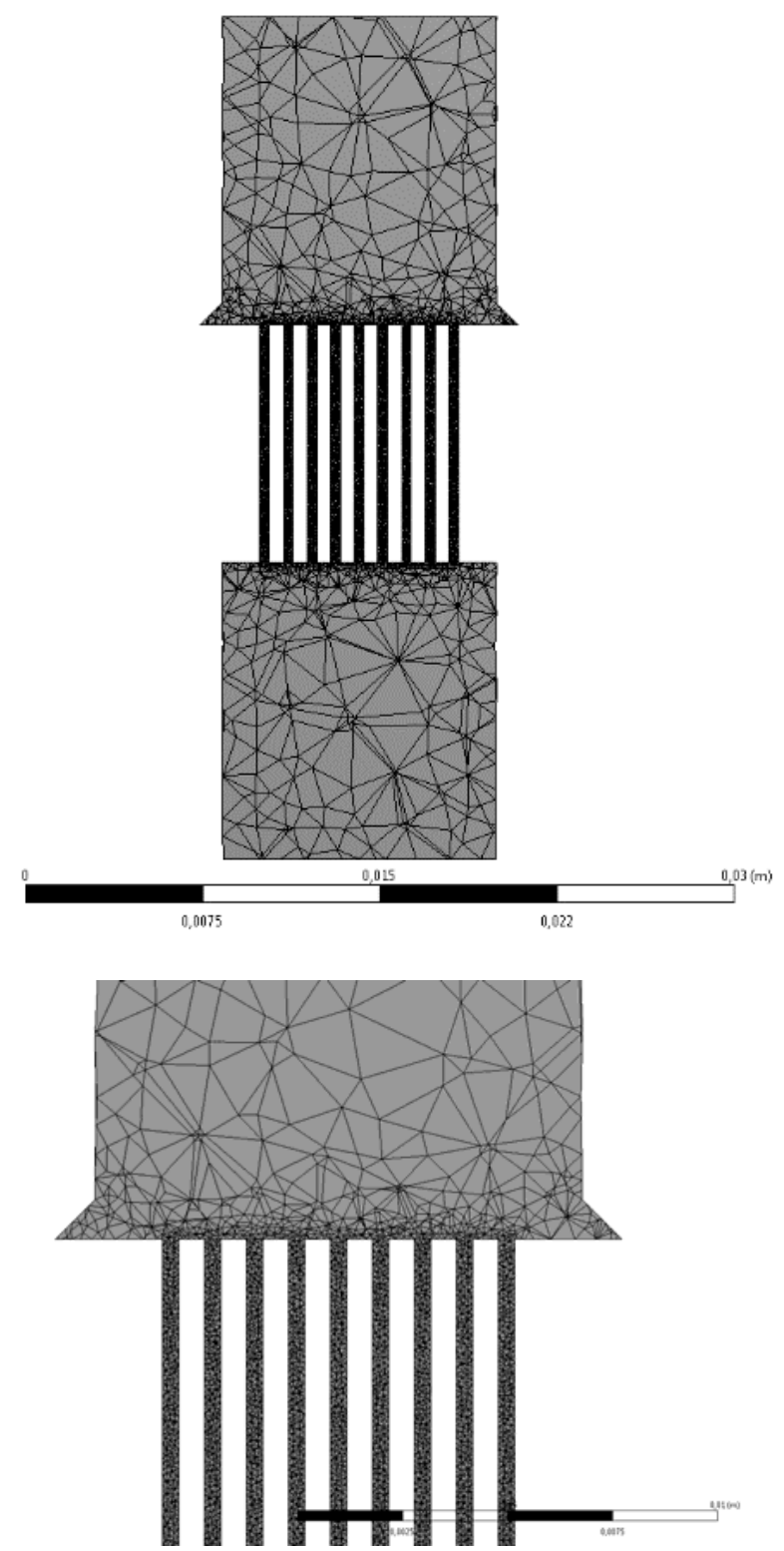

Fig. 3. The view of a computational grid, fluid filling the valve.

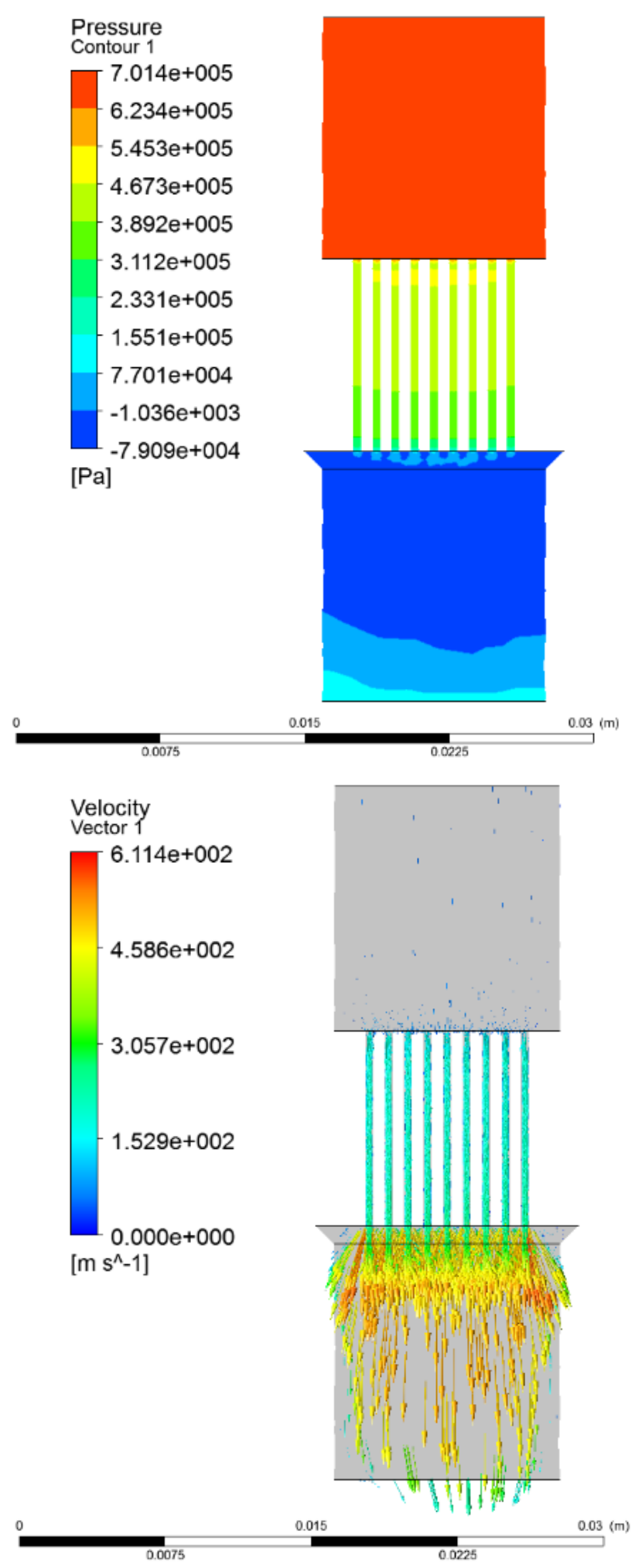

Fig. 4. Sectional view of the volume of valve slots, a) distribution of speed rectors, b) pressure distribution of the fluid. 

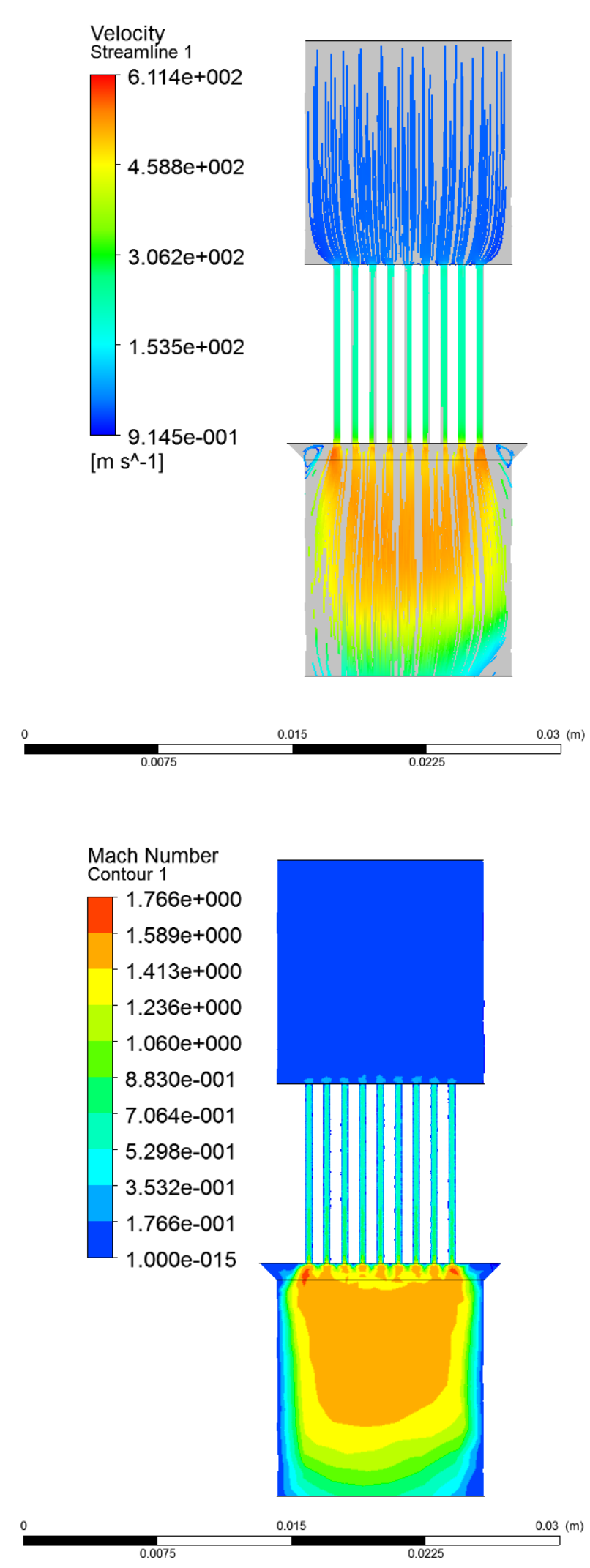

Fig. 5. Sectional view of the volume of valve slots, a) distribution of streamline, b) distribution of Mach number.

The modelling studies have set the maximum mass flow rate on the road $1-2$ amounting to $0.0343 \mathrm{~kg} / \mathrm{s}$.

\section{Conclusions}

The numerical methods of fluid mechanics used in modelling of the valve allowed to determine the flow parameters of the valve prototype in the initial phase of the design concerning the slots of a mobile insert. The use of numerical methods CFD can confirm the constructive assumptions and avoid time-consuming and expensive bench studies. The presented prototype of the valve has a number of features that distinguish it from the produced proportional valves. The analysis of the market of pneumatics and patent solutions shows the lack of a solution that would enable to obtain flows through the flow windows of the valve above 0.0343 $\mathrm{kg} / \mathrm{s}$ and the response time above $120 \mathrm{~Hz}$. The slotted structure of separation plates of the valve allows to accumulate the flow from individual windows and thus to construct the valve with so far unattainable flows of thousands litres per minute. In addition, the innovative structure of flow windows makes it possible to design valves of any flows adjusted to technological processes.

This solution is protected by patent PL $213836 \mathrm{~B} 1$.

This research was supported in part by PLGrid Infrastructure.

\section{References}

1. J.E. Takosoglu, (Acad Sci Czech Republic, Inst Thermomechanics, Dolejskova 5, Prague 8, 182 00, Czech Republic, 2016).

2. J.E. Takosoglu, P.A. Laski, S. Blasiak, G. Bracha, D. Pietrala, Measurement $\{\&\}$ Control. 49 (2) (2016), 62-71.

3. D.S. Pietrala, (Acad Sci Czech Republic, Inst Thermomechanics, Dolejskova 5, Prague 8, 182 00, Czech Republic, 2016).

4. J. Zwierzchowski, (Acad Sci Czech Republic, Inst Thermomechanics, Dolejskova 5, Prague 8, 182 00, Czech Republic, 2016).

5. S. Blasiak, C. Kundera, Procedia Engineering. 39 (2012), 315-326.

6. S. Blasiak, A. Pawinska, International Journal of Heat and Mass Transfer. 90 (2015), 710-718.

7. G.F. Bracha, (Acad Sci Czech Republic, Inst Thermomechanics, Dolejskova 5, Prague 8, 182 00, Czech Republic, 2016).

8. M. Blasiak, R. Kotowski, Przeglad Elektrotechniczny. 85 (12) (2009), 40-43.

9. D. Janecki, J. Zwierzchowski, (Imeko, Po Box 457, H-1371 5 Budapest, Hungary, 2009). 